\title{
Transgressive segregation of isoflavone contents under the control of four QTLs in a cross between distantly related soybean varieties
}

\author{
Takanori Yoshikawa ${ }^{1,2}$, Yutaka Okumoto*1), Daisuke Ogata ${ }^{1)}$, Takashi Sayama ${ }^{1,3)}$, Masayoshi Teraishii ${ }^{1)}$, \\ Masakazu Terai ${ }^{4}$, Toshiya Toda ${ }^{4}$, Katsushige Yamada ${ }^{4}$, Kazuhiro Yagasaki ${ }^{5}$, Naohiro Yamada ${ }^{5,6}$, \\ Takuji Tsukiyama', Toshiaki Yamada ${ }^{1)}$ and Takatoshi Tanisaka ${ }^{1)}$ \\ 1) Graduate School of Agriculture, Kyoto University, Oiwake, Kitashirakawa, Sakyo, Kyoto 606-8502, Japan \\ 2) Graduate School of Agriculture and Life Science, The University of Tokyo, 1-1-1 Yayoi, Bunkyo, Tokyo 113-8657, Japan \\ 3) National Agricultural Research Center for Hokkaido Region, 1 Hitsujigaoka, Toyohira, Sapporo, Hokkaido 062-8555, Japan \\ 4) Fujicco Co., Ltd., 6-13-4 Nakamachi, Minatojima, Chuo, Kobe, Hyogo 650-8558, Japan \\ 5) Nagano Vegetable and Ornamental Crops Experiment Station, 1066-1 Aza-tokoo, Oaza-soga, Shiojiri, Nagano 399-6461, Japan \\ 6) National Institute of Crop Science, 2-1-18 Kannondai, Tsukuba, Ibaraki 305-8518, Japan
}

\begin{abstract}
Soybean (Glycine max (L) Merr.) isoflavones have attracted considerable attention for their diverse effects on human health. To determine the genetic factors that contribute to the high isoflavone contents of the soybean varieties 'Peking' and 'Tamahomare', we conducted QTL analyses for the total content of daidzein derivatives (DAC), genistein derivatives (GEC) and glycitein derivatives (GLC), and for the total content of isoflavones (TIC) using recombinant inbred lines (RILs) derived from the cross between 'Peking' and 'Tamahomare'. Ninety six RILs were planted in Kyoto, Osaka and Nagano in 2003 and in Osaka and Nagano in 2004. Transgressive segregation for TIC was detected in all the environments tested. Composite interval mapping for TIC revealed four QTLs: qIso1, qIso2, qIso3 and qIso4, located on LG-A1 (Chr.5), LG-A2 (Chr.8), LG-C1 (Chr.4) and LG-D2 (Chr.17), respectively. The high-isoflavone alleles were derived from Peking at qIsol and qIso4 and from Tamahomare at qIso2 and qIso3. Several other groups have already reported the former two QTLs, but qIso2 and qIso3 are new discoveries. Our results indicate that the large variation in TIC measurements observed in the RILs could have resulted from the combined effects of alleles at the four QTLs derived from distantly related varieties.
\end{abstract}

Key Words: Glycine $\max (\mathrm{L})$ Merr., isoflavone content, QTL, recombinant inbred line, transgressive segregation.

\section{Introduction}

Isoflavones are synthesized predominantly in legumes, where they act as antifungal compounds (Rivera-Vargas et al. 1993), attractors of Rhizobial bacteria (van Rhiji et al. 1995), and inducers of nod (nodulation) gene expression to initiate nitrogen-fixing root nodule formation (Pueppke 1996). In the chickpea (Cicer arietinum L.), isoflavone malonylglycosides are located exclusively in the vacuole, and malonylesterase, which is involved in the ester hydrolysis of isoflavones, is associated with the vacuolar membrane (Barz et al. 1992, Mackenbrock et al. 1992).

Isoflavones possess a structure similar to that of estradiol, showing conformational binding to estrogen receptors (Setchell 2001). Epidemiologic research has indicated that women ingesting high amounts of soy products are less likely to develop cardiovascular disease, breast and uterine can-

Communicated by J. Abe

Received April 8, 2010. Accepted July 22, 2010.

*Corresponding author (e-mail: okumoto3@kais.kyoto-u.ac.jp) cer, and menopausal symptoms than those consuming western diets containing fewer soy products (Lissin and Cooke 2000). Other recent studies have suggested that isoflavones might possess other health-promoting activities including chemoprevention of osteoporosis and cardiovascular disease (Alekel et al. 2000, MerzDemlow et al. 2000, Uesugi et al. 2001). As a result, soybean (Glycine max Merr.) seed has attracted considerable attention as a potential dietary supplement due to its high concentration of isoflavones (Wang et al. 1994b). On the other hand, several researchers have suggested that isoflavones also exert negative biological effects, possibly resulting in thymic and immune abnormalities among infants who are fed soy-based infant formulas (Setchell et al. 1997, Fitzpatrick 1998, Yellayi et al. 2002). Thus it is important for human health and food safety agencies as well as soy-based food processing manufacturers to regulate isoflavone content in soybean seed.

The isoflavones that occur in soybean seeds can be categorized into three groups: viz., daidzein derivatives [daidzein, daidzin, acetyldaidzin and malonyldaidzin], genistein derivatives [genistein, genistin, acetylgenistin and 
malonylgenistin] and glycitein derivatives [glycitein, glycitin, acetylglycitin and malonylglycitin]. The isoflavone content in soybean seed is influenced by diverse environmental factors including temperature during the seedripening period (Tsukamoto et al. 1995), nutritional status of the soil (Vyn et al. 2002, Landini et al. 2002), fungal infection (Graham and Graham 1996, Dixon et al. 1995) and UV irradiation (Dixon et al. 1995). In contrast to these environmental factors, little information has been obtained about the genetic regulation mechanisms of the isoflavone content.

Previous reports (Meksem et al. 2001, Kassem et al. 2004, Primomo et al. 2005, Kassem et al. 2006, Zeng et al. 2009) have identified QTLs that control the content of daidzein derivatives on linkage groups (LG, hereafter) A1, F, $\mathrm{H}, \mathrm{I}, \mathrm{K}, \mathrm{M}$ and N. QTLs controlling the content of genistein derivatives have been identified on LG-A2, C2, F, G, H and M. QTLs controlling the content of glycitein derivatives have been identified on LG-B1, D1a, F, G, H, I, J, M, N and O. In addition, several QTLs that regulate the total isoflavone content as well as the content of all isoflavone groups have been identified. Primomo et al. (2005) detected a significant QTL controlling the total isoflavone content on LG-M, which is responsible for $25.5 \%$ of the phenotypic variation that occurs under a given set of environmental conditions. Likewise, Zeng et al. (2009) identified significant QTLs controlling the total isoflavone content on LG-D2, F, G, O and M.

In the present study, prominent transgressive segregants with extremely high total isoflavone content were found among the recombinant inbred lines derived from the cross between the varieties referred as 'Peking' and 'Tamahomare' (PT-RILs). 'Peking' is a landrace originating in China, whereas 'Tamahomare' is a typical Japanese variety bred from a cross combination between a Japanese breeding line and a variety from the USA known as 'Lee'. The present study aimed to disclose the genetic factors that confer the high rate of transgressive segregation seen in PT-RILs. The effects of those factors on isoflavone content and their interactions were also clarified.

\section{Materials and Methods}

\section{Genetic variation in isoflavone content}

Sixty soybean varieties originating from several areas of Japan, including 'Tamahomare' and the Chinese landrace 'Peking', were grown in Kyoto in 2003 to evaluate variation in total isoflavone content among Japanese soybean varieties. Four different seed coat types were present among the varieties: 20 varieties had yellow seed coats, 10 had red seed coats, 6 had brown seed coats and 24 had black seed coats. The isoflavone contents of all varieties were determined using simplified methods (Yoshikawa et al. 2009). Seeds were sown on 18 June in 2003. $\mathrm{N}, \mathrm{P}_{2} \mathrm{O}_{5}$ and $\mathrm{K}_{2} \mathrm{O}$ were applied as basal fertilizers in quantities of 20,60 and $70 \mathrm{~kg} / \mathrm{ha}$, respectively. Plant spacing was $10 \times 30 \mathrm{~cm}$.

\section{PT-RILS}

Ninety-six RILs $\left(\mathrm{F}_{8: 9}\right)$, derived from the cross between the 'Peking' and 'Tamahomare' soybean varieties were used in this study. They were planted together with their parental varieties in fields at three locations: Kyoto (average temperature during maturation: $23.9^{\circ} \mathrm{C}$, latitude: $35^{\circ} 01^{\prime} \mathrm{N}$, elevation above sea level: $90 \mathrm{~m}$ ), Osaka (average temperature during maturation: $26.4^{\circ} \mathrm{C}$, latitude: $34^{\circ} 51^{\prime} \mathrm{N}$, elevation above sea level: $10 \mathrm{~m}$ ) and Nagano (average temperature during maturation: $20.5^{\circ} \mathrm{C}$, latitude: $30^{\circ} 07^{\prime} \mathrm{N}$, elevation above sea level: $750 \mathrm{~m}$ ) in Japan. This field experiment was conducted using a randomized block design with two replications in 2003 and 2004. Each plot contained 30 plants. In 2003, seeds were sown on 18 June in Kyoto, on 5 June in Osaka and on 29 May in Nagano; in 2004, seeds were sown on 3 June in Osaka and on 2 June in Nagano. Fertilizer application and plant spacing were carried out as described in the previous subsection. Air temperature in the field was recorded at intervals of 1 hour, and the daily mean temperature was calculated. Flowering date (R1) and maturating date (R8) were also observed for all PT-RILs and their parental varieties in all three locations. The flowering times and maturating times were calculated as the number of days from sowing (S) to flowering (R1) and the number of days from flowering (R1) to maturing (R8), respectively.

\section{Measurement of isoflavone content}

HPLC methods: Matured seeds of all the PT-RILs were harvested and air-dried in a greenhouse for at least 1 week. After the seed coats were peeled manually, ca. $5 \mathrm{~g}$ of seeds from each plot was milled with a Multi-beads shocker (MB400U; Yasui Kikai, Co., Ltd., Osaka, Japan). Two g of the resulting seed powder was dried at $90^{\circ} \mathrm{C}$ for 24 hours, and its water content was measured. The water content of the seed powder was calculated by subtracting the seed powder's dry weight from its fresh weight. The remaining powder (ca. $3 \mathrm{~g}$ ) was used for isoflavone analysis, which was conducted according to the method of Kudou et al. (1991). Twenty-five $\mathrm{ml}$ of $70 \% \mathrm{EtOH}$ was added to the seed powder, and the mixture was shaken at room temperature for $30 \mathrm{~min}$. After centrifugation, the supernatant was collected. This extraction procedure was repeated three times, and $70 \% \mathrm{EtOH}$ was added to the collected supernatant up to a volume of 100 $\mathrm{ml}$. After filtration with a $0.45-\mu \mathrm{m}$ dismic filter (Advantec Toyo Kaisha, Ltd., Tokyo, Japan), $10 \mu$ of the isoflavone extracts were analyzed with a reverse-phase HPLC system (Shimadzu Co. Ltd., Kyoto, Japan) equipped with a YMCPack ODS-AM303 (4.6×250 mm; YMC, Co., Ltd., Kyoto, Japan). The mobile phase of the HPLC consisted of $15 \%$ and $35 \%$ acetonitrile solution containing $0.1 \%$ acetic acid. Isoflavones were separated using a liner gradient from $15 \%$ to $35 \%$ acetonitrile solution at a flow rate of $1.0 \mathrm{ml} / \mathrm{min}$ over $50 \mathrm{~min}$ (Kudou et al. 1991). Isoflavones were detected at $254 \mathrm{~nm}$ at $35^{\circ} \mathrm{C}$, and each isoflavone component was identified based on the retention time of pure samples provided by Fujicco Co., Ltd., Kobe, Japan. 
Simplified method: Matured seeds of 60 varieties were powdered and isoflavone content was measured according to a simplified method (Yoshikawa et al. 2009). First, isoflavones were extracted from the ca. $3 \mathrm{~g}$ of seed powder of each variety with $25 \mathrm{ml}$ of $70 \% \mathrm{EtOH}$. The concentration $(\mathrm{mg} / \mathrm{ml})$ of isoflavones was estimated based on the absorbance of 260-nm wavelength (A260) of crude extract measured with a spectrophotometer (Shimadzu Co., Ltd., Kyoto, Japan). Isoflavones consist of four derivatives for each of three different isoflavone groups, and each derivative exhibits a unique absorption coefficient at a specific wavelength of UV light such as $260 \mathrm{~nm}$. In the case of soybean varieties with nearly constant relative contents of twelve different isoflavones, however, we could roughly estimate the total isoflavone contents with the A260 of crude extract. The total isoflavone contents of ten selected varieties were determined using the HPLC system in order to generate a standard curve in estimating the total isoflavone content (TIC) based on A260. Correlation between TIC and A260 was as high as 0.96 (significant at the 0.01 level). The TIC $(\mathrm{mg} / \mathrm{g})$ of each variety was estimated according to the linear regression relationship $(Y=\mathrm{A} 260 \times 0.2514 \times 25 / X)$, where $Y$ and $X$ indicated TIC ( $\mathrm{mg} / \mathrm{g})$ and the sample's dry weight $(\mathrm{g})$, respectively. A260 was converted to the concentration of isoflavones $(\mathrm{mg} / \mathrm{ml})$ through multiplication by 0.2514 . Finally, the total isoflavone contents were determined by multiplying the concentration of isoflavones $(\mathrm{mg} / \mathrm{ml})$ by the quantity of extraction buffer $(25 \mathrm{ml})$. In this study, all isoflavone contents were recorded on a dry-weight basis.

Analyses of variance: Calculations of the expected mean squares and sources of variations of isoflavone derivatives for complete randomized block experiments combining three locations and two years (Kyo03, Osk03, Nag03, Osk04, Nag04) were conducted as described by McIntosh (1983). Mean squares of environments (E), replications (R), lines (L), interaction between environment and line (EL) and error (e) were defined as M1 $=\sigma_{e}^{2}+t \sigma_{R}^{2}+r t \sigma_{E}^{2}, \mathrm{M} 2=\sigma_{e}^{2}$ $+t \sigma_{R}^{2}, \mathrm{M} 3=\sigma_{e}^{2}+r \sigma_{E L}^{2}+r l \sigma_{L}^{2}, \mathrm{M} 4=\sigma_{e}^{2}+\sigma_{E L}{ }^{2}$ and M5 $=\sigma_{e}^{2}$, respectively $(r=$ Number of replications, $l=$ Number of environments, and $t=$ Number of lines). Effects of environment $(\mathrm{E})$, line $(\mathrm{L})$ and $\mathrm{EL}$ interaction were tested with the F-ratios of M1/M2, M3/M4 and M4/M5, respectively. Broad sense heritability $\left(h^{2}\right)$ for mean values over environments was calculated from components of variance: $h^{2}=\sigma_{\mathrm{g}}{ }^{2} /\left(\sigma_{\mathrm{g}}{ }^{2}\right.$ $\left.+\sigma_{\mathrm{g} \times \mathrm{e}}{ }^{2} / 1+\sigma_{e}{ }^{2} / \mathrm{lr}\right)$. Variance components of $\sigma_{\mathrm{g}}{ }^{2}, \sigma_{\mathrm{g} \times \mathrm{e}}{ }^{2}$ and $\sigma_{\mathrm{e}}{ }^{2}$ were estimated from $\sigma_{L}^{2}, \sigma_{E L}^{2}$ and $\sigma_{e}^{2}$, respectively.

\section{QTL analysis of the isoflavone contents}

The linkage map of the PT-RIL population reported by Sayama et al. (2009) consists of 344 simple sequence repeat (SSR) markers and three conventional markers, Inhibitor (I), pubescence color $(T)$ and flower color (W1) loci, covering $2652.5 \mathrm{cM}$. The average distance between markers was $7.6 \mathrm{cM}$. QTL analysis for isoflavone content was conducted using Windows QTL Cartographer ver. 2.5 (Zeng et al. 1993, 1994). In the QTL analysis, we conducted the standard model of composite interval mapping (CIM) (forward regression method). To obtain the optimum thresholds for the presence of QTL, we conducted a permutation test with 1,000 repetitions for each trait at the significance level of 0.05 . QTL was then tested at 0.5 -cM interval. CIM reports a likelihood ratio test (LTR) statistic, which can be converted to a logarithm of the odd (LOD) score by means of the following formula: $\mathrm{LOD}=\mathrm{LRT} / 2 \times \ln (10)$. The LOD score peak was used to estimate the most likely position of each QTL on the linkage map. The results of the permutation test indicated that most thresholds for LOD score were greater than 3.7. When we applied this criterion to our search for QTL, however, we detected only one QTL for most traits under all environmental conditions. It is therefore possible that most potential QTLs are undetectable at this threshold. Accordingly, we also examined minor QTLs with LOD score values greater than 3.0. To evaluate the effects of interactions among detected QTLs, multiple regression analysis was conducted (Watanabe et al. 2004). To calculate those parameters, we determined the genotype of each QTL based on the nearest marker. An example of the equation for two-factor multiple regression analysis for two QTLs is as follows:

$$
y_{i}=\mu+x_{1 i} a+x_{2 i} b+x_{1 i} x_{2 i} c+\varepsilon_{i}
$$

where $y_{i}$ : total isoflavone content (TIC) of each line $i, \mu$ : mean of TIC, $a$ : additive effect of QTL-1, $b$ : additive effect of QTL-2, $c$ : effect of interaction between QTL-1 and QTL$2, \varepsilon_{i}$ : error. $x_{1 i}$ and $x_{2 i}$ denote the variables based on the genotypes of QTL1 and QTL2 being 1 and -1 for a Peking allele and a Tamahomare allele, respectively. All calculations were conducted using $\mathrm{R}$ software (R Foundation for Statistical Computing, Vienna, Austria, version 2.9).

\section{Results}

\section{Genetic variation in isoflavone content}

The total isoflavone contents (TIC) of the 60 soybean varieties analyzed in this study, as estimated by the simplified method, exhibited a wide distribution, from 1.07 to $6.92 \mathrm{mg} /$ g (Fig. 1). While the mean TIC was estimated at $3.23 \mathrm{mg} / \mathrm{g}$, the TIC of Tamahomare and Peking were estimated at $6.43 \mathrm{mg} / \mathrm{g}$ and $5.18 \mathrm{mg} / \mathrm{g}$, respectively. This indicates that the TICs of both parental varieties of the PT-RILs are higher than those of most other Japanese soybean varieties. When soybean varieties were grouped according to seed coat color, the average TIC values of the yellow seed coat group (Y), the red-brown seed coat group (R), the brown seed coat group $(\mathrm{Br})$ and the black seed coat group $(\mathrm{BL})$ were $3.47 \mathrm{mg} /$ $\mathrm{g}, 3.20 \mathrm{mg} / \mathrm{g}, 2.67 \mathrm{mg} / \mathrm{g}$ and $3.39 \mathrm{mg} / \mathrm{g}$, respectively. Thus we could find no significant difference in terms of TIC among the four groups based on seed coat color.

\section{Phenotypic variation among the RILs}

In 2003, all PT-RILs were grown in Kyoto (Kyo03), Osaka (Osk03) and Nagano (Nag03). In 2004, they were 


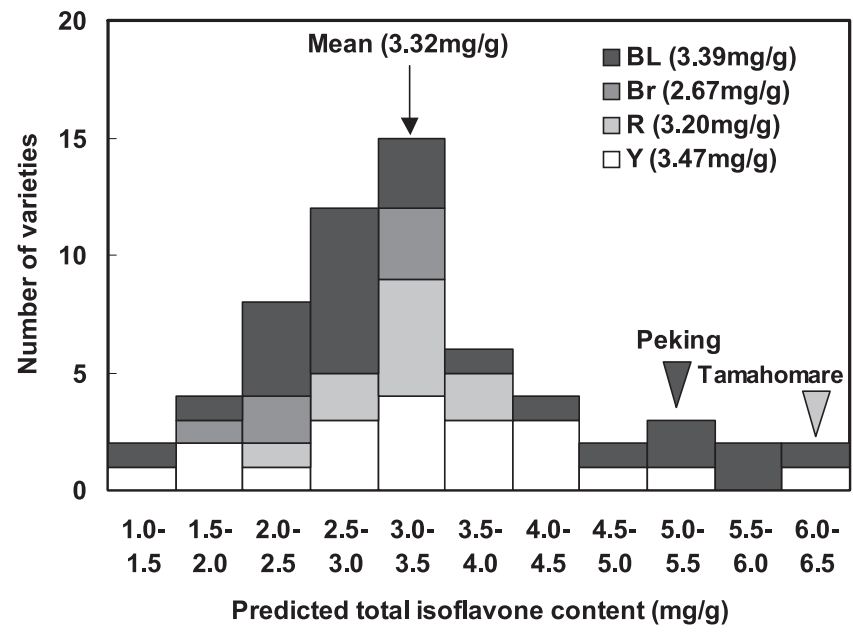

Fig. 1. Frequency distribution of the total isoflavone content $(\mathrm{mg} / \mathrm{g})$ of 60 soybean varieties, as evaluated by the simplified isoflavone quantification method. These varieties were classified according to seed coat color (BL: Black, Br: Brown, R: Red, Y: Yellow). The bracketed values are the mean total isoflavone content values for each seed coat group (BL, Br, R, and $\mathrm{Y}$ ). The arrow indicates the mean isoflavone content of the soybeans when all 60 varieties were considered together, and the arrowheads indicate the total isoflavone content of Peking (P: $5.17 \mathrm{mg} / \mathrm{g}$ ) and Tamahomare (T: $6.42 \mathrm{mg} / \mathrm{g}$ ).

grown in Osaka (Osk04) and Nagano (Nag04). In the Kyo03, Osk03 and Osk04 samples, the TIC values of Peking and Tamahomare were almost identical. In the samples grown in Nagano, however, the TIC of Tamahomare was much lower than that of Peking in both years, due to the low daidzein derivative values in Tamahomare. In all locations and years, the PT-RIL population showed transgressive segregation in TIC, total content of daidzein derivatives (DAC), total content of genistein derivatives (GEC) and total content of glycitein derivatives (GLC) values (Table 1). TIC values of parental varieties were almost identical to the mean TIC values of PT-RILs, whereas the maximum value of PT-RILs for TIC was almost ten times larger than the minimum value. Frequency distributions of TIC values among the PT-RILs were not significantly different from the normal distribution, and the values were almost evenly distributed around those of the parental varieties. The same tendency was observed in the frequency distributions of DAC, GEC and GLC values among the PT-RILs. These data indicate that both varieties carry independent genetic factors that increase the isoflavone content of all three groups, i.e., DAC, GEC and GLC. The effects on TIC, DAC, GEC and GLC of interactions between genotype and environment $(\mathrm{L} \times \mathrm{E})$ were very small compared to the main genotypic and environmental effects. In most of the previous reports on QTLs for TIC (Meksem et al. 2001, Kassem et al. 2004, Primomo et al. 2005, Kassem et al. 2006, Zeng et al. 2009), the effects of the interaction between genotype and environment were reported to be quite large, and the estimated values of heritability for isoflavone contents were no greater than $40 \%$. In this study, however, the values of observed broad sense heritability of TIC, DAC, GEC and GLC were as high as 90\% (Table 2). This indicates that the most of the variation among PT-RILs in terms of isoflavone content could be due to genetic factors. In the PT-RIL population, DAC and GEC showed strong positive correlations with TIC. The correlation coefficients of TIC and DCA observed in Kyo03, Osk03, Nag03, Osk04 and Nag04 were 0.96, 0.98, 0.94, 0.96 and 0.95, respectively. All of these were highly significant ( $\alpha=0.001)$. The correlation coefficients of TIC and GEC were also highly significant: in Kyo03, Osk03, Nag03, Osk04 and Nag04 they were $0.95,0.96,0.94,0.95$ and 0.95 , respectively. In contrast, the correlation coefficients of TIC and GLC were lower in all locations and years: in Kyo03, Osk03, Nag03, Osk04 and Nag04 they were $0.75,0.53,0.59,0.64$ and 0.53 , respectively. Furthermore, in all locations and years, the sum of DAC and GEC accounted for more than $80 \%$ of the TIC, while GLC accounted for only around $10 \%$ of the TIC. As Wang et al. (1994a) have shown, the wide variation observed in TIC among PT-RILs was mainly caused by the wide variation in DAC and GEC.

To evaluate the effects of temperature on isoflavone content, we calculated average daily mean temperature in the period from R1 (flowering) to R8 (seed maturing) for each line in each location. Total isoflavone content (TIC) and average daily mean temperature during the maturation period (AMT) exhibited weak negative correlation in all locations and years (Fig. 2), but wide variation in TIC was observed among lines with the same AMT values, clearly indicating that temperature was not the main factor responsible for determining the TIC. In 2003, the mean TIC of PT-RILs was lowest in Osaka, intermediate in Nagano, and highest in Kyoto, while the mean AMT of PT-RILs was lowest in Nagano $\left(20.5^{\circ} \mathrm{C}\right)$, intermediate in Kyoto $\left(23.9^{\circ} \mathrm{C}\right)$, and highest in Osaka $\left(26.4^{\circ} \mathrm{C}\right)$. In addition to this, the mean TIC of PT-RILs in Osaka was not significantly different from that in Nagano, even though the mean AMT in Nagano was lower than that in Osaka by more than five degrees. Thus the negative correlation between TIC and AMT observed in the RILs may have resulted not from any underlying physiological association but rather from indirect effects such as linkages.

\section{QTL analysis for isoflavone content}

QTL analysis on the RIL populations grown in Kyoto, Osaka and Nagano in 2003 revealed several QTLs associated with TIC, DAC, GEC and GLC (Table 3). The QTL with the largest effects on TIC was detected on LG-A2 (Chr. 8) (KT_1, OT_2 and NT_1) in all three locations. The allele at this QTL that is derived from Tamahomare is a high-isoflavone allele. The same QTL was also detected for DAC $\left(K D \_1, O D \_2, N D \_1\right), \operatorname{GEC}\left(K G \_1, O G_{-} 1, N G \_1\right)$ and GLC ( $K G l$ l, OGl 2 and $N G l$ l). Tamahomare also harbors another high-isoflavone allele at a QTL located on LG-C1 (Chr. 4). This QTL was associated with TIC and GEC ( $\left.K T \_2, K G \_2\right)$ in Kyoto, and with GEC (OG_2) in Osaka. In addition to these QTLs, another two QTLs were 
Table 1. Means and ranges of the PT-RIL population and parental varieties for the total isoflavones content (TIC), total daidzein derivatives content (DAC), total genistein derivatives content (GEC) and total glycitein derivatives content (GLC), flowering time (S-R1), and maturing time (R1-R8) planted in Kyoto, Osaka, and Nagano in 2003 and 2004

\begin{tabular}{|c|c|c|c|c|c|c|}
\hline \multirow{2}{*}{ Environment } & \multirow{2}{*}{ Trait $^{a}$} & \multicolumn{2}{|c|}{ Parental varieties ${ }^{b}$} & \multicolumn{3}{|c|}{ RIL population } \\
\hline & & Peking & Tamahomare & Minimum & Maximum & Mean $^{c}$ \\
\hline \multirow[t]{6}{*}{ Kyoto 2003} & TIC (mg/g) & $6.14 \pm 0.95$ & $6.51 \pm 0.40$ & 2.08 & 11.03 & $6.44 \pm 2.098$ \\
\hline & $\mathrm{DAC}(\mathrm{mg} / \mathrm{g})$ & $3.04 \pm 0.55$ & $2.45 \pm 0.22$ & 0.66 & 6.27 & $2.88 \pm 1.173$ \\
\hline & GEC (mg/g) & $2.36 \pm 0.34$ & $3.34 \pm 0.19$ & 1.01 & 4.65 & $2.92 \pm 0.873$ \\
\hline & GLC (mg/g) & $0.73 \pm 0.05$ & $0.72 \pm 0.01$ & 0.23 & 1.06 & $0.63 \pm 0.179$ \\
\hline & S-R1 (days) & $46.2 \pm 0.3$ & $43.9 \pm 0.1$ & 36.5 & 67.0 & $48.6 \pm 6.28$ \\
\hline & R1-R8 (days) & $60.3 \pm 0.6$ & $82.0 \pm 1.5$ & 59.7 & 92.9 & $72.9 \pm 6.38$ \\
\hline \multirow[t]{6}{*}{ Osaka 2003} & TIC (mg/g) & $4.25 \pm 0.02$ & $4.79 \pm 0.33$ & 0.90 & 9.99 & $4.73 \pm 2.111$ \\
\hline & $\mathrm{DAC}(\mathrm{mg} / \mathrm{g})$ & $2.02 \pm 0.02$ & $2.00 \pm 0.21$ & 0.19 & 5.45 & $2.19 \pm 1.172$ \\
\hline & GEC (mg/g) & $1.61 \pm 0.05$ & $2.42 \pm 0.12$ & 0.45 & 4.14 & $2.09 \pm 0.914$ \\
\hline & GLC (mg/g) & $0.62 \pm 0.05$ & $0.38 \pm 0.00$ & 0.14 & 0.83 & $0.44 \pm 0.155$ \\
\hline & S-R1 (days) & $45.5 \pm 1.5$ & $46.0 \pm 0.0$ & 37.5 & 76.0 & $51.6 \pm 7.85$ \\
\hline & R1-R8 (days) & $64.5 \pm 1.5$ & $110.0 \pm 0.0$ & 67.0 & 124.5 & $90.2 \pm 10.85$ \\
\hline \multirow[t]{6}{*}{ Nagano 2003} & TIC (mg/g) & $6.95 \pm 0.63$ & $4.85 \pm 0.13$ & 2.86 & 9.59 & $5.87 \pm 1.589$ \\
\hline & $\mathrm{DAC}(\mathrm{mg} / \mathrm{g})$ & $3.58 \pm 0.41$ & $1.97 \pm 0.06$ & 0.92 & 4.57 & $2.56 \pm 0.856$ \\
\hline & GEC (mg/g) & $2.87 \pm 0.29$ & $2.59 \pm 0.10$ & 1.11 & 4.55 & $2.82 \pm 0.761$ \\
\hline & GLC (mg/g) & $0.51 \pm 0.07$ & $0.29 \pm 0.09$ & 0.22 & 0.84 & $0.49 \pm 0.124$ \\
\hline & S-R1 (days) & $66.8 \pm 0.3$ & $65.5 \pm 0.0$ & 60.0 & 91.0 & $71.6 \pm 7.00$ \\
\hline & R1-R8 (days) & $61.8 \pm 1.3$ & $89.8 \pm 0.3$ & 61.5 & 93.5 & $77.7 \pm 6.75$ \\
\hline \multirow[t]{6}{*}{ Osaka 2004} & TIC (mg/g) & $4.21 \pm 0.21$ & $4.17 \pm 0.57$ & 1.41 & 8.69 & $4.92 \pm 1.719$ \\
\hline & DAC (mg/g) & $2.13 \pm 0.17$ & $1.61 \pm 0.31$ & 0.47 & 4.60 & $2.23 \pm 0.988$ \\
\hline & GEC (mg/g) & $1.62 \pm 0.10$ & $2.12 \pm 0.23$ & 0.70 & 3.70 & $2.17 \pm 0.700$ \\
\hline & GLC (mg/g) & $0.46 \pm 0.06$ & $0.44 \pm 0.02$ & 0.15 & 0.88 & $0.51 \pm 0.161$ \\
\hline & S-R1 (days) & $45.5 \pm 0.5$ & $46.5 \pm 0.5$ & 36.5 & 82.0 & $52.5 \pm 8.89$ \\
\hline & R1-R8 (days) & $72.0 \pm 2.0$ & $100.5 \pm 1.5$ & 72.5 & 108.0 & $87.6 \pm 8.42$ \\
\hline \multirow[t]{6}{*}{ Nagano 2004} & TIC (mg/g) & $6.13 \pm 0.39$ & $3.96 \pm 0.66$ & 1.71 & 8.23 & $4.90 \pm 1.468$ \\
\hline & DAC (mg/g) & $3.27 \pm 0.20$ & $1.54 \pm 0.24$ & 0.66 & 4.67 & $2.24 \pm 0.790$ \\
\hline & GEC (mg/g) & $2.46 \pm 0.17$ & $2.14 \pm 0.38$ & 0.88 & 3.99 & $2.33 \pm 0.689$ \\
\hline & GLC (mg/g) & $0.39 \pm 0.02$ & $0.28 \pm 0.03$ & 0.12 & 0.61 & $0.32 \pm 0.107$ \\
\hline & S-R1 (days) & $59.0 \pm 0.0$ & $58.5 \pm 0.5$ & 49 & 88 & $64.4 \pm 7.78$ \\
\hline & R1-R8 (days) & $64.0 \pm 1.0$ & $93.5 \pm 3.5$ & 65 & 90 & $78.4 \pm 4.81$ \\
\hline
\end{tabular}

${ }^{a}$ DAC, GEC, GLC, and TIC were calculated as follows: DAC; the total content of daidzein, daidzin, acetyldaidzin, and malonyldaidzin, GEC; the total content of genistein, genistin, acetylgenistin, and malonylgenistin, GLC; the total content of glycitein, glycitin, acetylglycitin, and malonylglycitin, TIC; the total content of DAC, GEC, and GLC, S-R1; Number of days from sowing to flowering, R1-R8; Number of days from flowering to maturing.

${ }^{b}$ Mean \pm SE.

${ }^{c}$ Mean \pm SD.

Table 2. Analysis of variance for the total isoflavones content (TIC), total daidzein derivatives content (DAC), total genistein derivatives content (GEC) and total glycitein derivatives content (GLC) in the PT-RIL population planted in Kyoto, Osaka, and Nagano in 2003 and 2004

\begin{tabular}{|c|c|c|c|c|c|c|c|c|}
\hline \multirow{2}{*}{ Factor } & \multicolumn{2}{|c|}{ TIC } & \multicolumn{2}{|c|}{ DAC } & \multicolumn{2}{|c|}{ GEC } & \multicolumn{2}{|c|}{$\overline{\text { GLC }}$} \\
\hline & $F$-value & probability & F-value & probability & $F$-value & probability & F-value & probability \\
\hline Environment (E) & 67.00 & $<0.001$ & 195.27 & $<0.001$ & 350.95 & $<0.001$ & 625.09 & $<0.001$ \\
\hline Lines (L) & 22.11 & $<0.001$ & 106.68 & $<0.001$ & 68.12 & $<0.001$ & 48.69 & $<0.001$ \\
\hline $\mathrm{L} \times \mathrm{E}$ & 4.22 & $<0.001$ & 4.15 & $<0.001$ & 3.67 & $<0.001$ & 2.71 & $<0.001$ \\
\hline$h^{2}$ & 0.95 & & 0.98 & & 0.94 & & 0.94 & \\
\hline
\end{tabular}

***; Statistically significant at 0.001 level.

$\mathrm{h}^{2}=\sigma_{\mathrm{g}}{ }^{2} /\left(\sigma_{\mathrm{g}}^{2}+\sigma_{\mathrm{g} \times \mathrm{e}}{ }^{2} / 1+\sigma_{\mathrm{e}}{ }^{2} / \mathrm{lr}\right) ; \sigma_{\mathrm{g}}{ }^{2}=\left(\mathrm{MS}_{\mathrm{L}}-\mathrm{MS}_{\mathrm{EL}}\right) / \mathrm{lr}, \sigma_{\mathrm{g} \times \mathrm{e}}{ }^{2}=\left(\mathrm{MS}_{\mathrm{EL}}-\mathrm{MS}_{\mathrm{e}}\right) / \mathrm{r}$.

1 and $\mathrm{r}$ : Number of environments and number of replications per environment. 

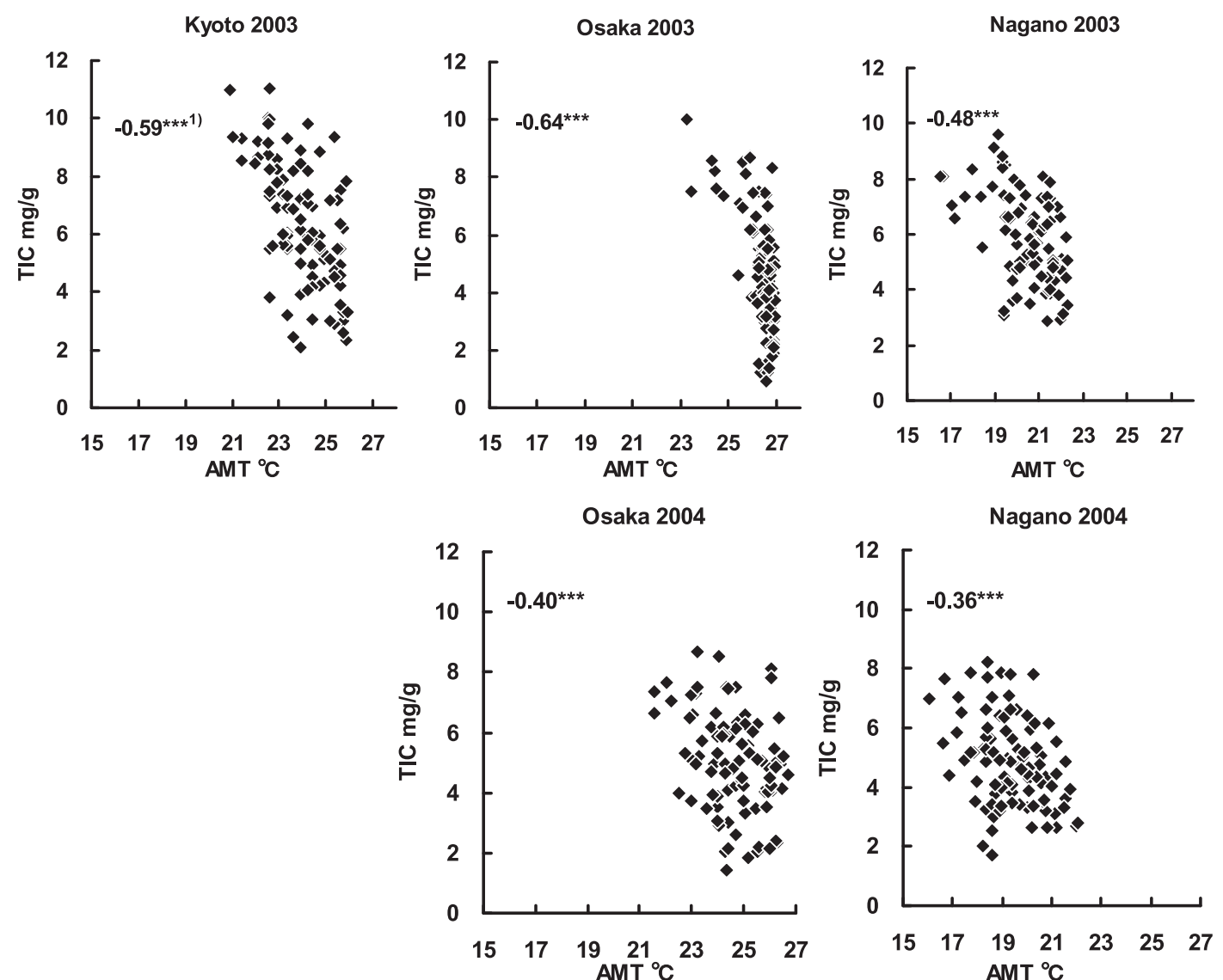

Fig. 2. Relationships between total isoflavone content (TIC) and average daily mean temperature during the maturation period (AMT) in the PTRIL population planted in Kyoto, Osaka and Nagano in 2003 and Osaka and Nagano in 2004. Correlation coefficient between TIC and AMT. ***: Significant at $0.1 \%$ level.

also detected on LG-A1 (Chr. 5) and LG-D2 (Chr. 17). In contrast to the former two QTLs, the high-isoflavone alleles at the latter two were derived from Peking. The QTL located on LG-A1 was associated with TIC (OT 1) and DAC $\left(O D \_1\right)$, and the QTL located on LG-D2 was associated with TIC $(K T$ 3) and GEC ( $K G$ 2). The QTL located on LG-B1 (Chr. 11) was observed to be associated with GLC (OGl2, $N G l$ 2). In conclusion, the QTLs located on LG-A2 in the vicinity of $I$ locus and the QTL located on LG-C1 in the vicinity of Satt190 were considered to be the QTLs contributing to the high isoflavone content of Tamahomare. As for Peking, the two QTLs contributing to its high isoflavone content were considered to be those located on LG-A1 in the vicinity of Sat_271 and those located on LG-D2 in the vicinity of Satt669. The QTL detected on LG-B1 in the vicinity of Satt197 might be the specific QTL for GLC.

In 2004, a QTL for TIC and all other isoflavone groups was detected on LG-A2 (OT_4,OD_4, OG_4, OGl_3, $N T \_2, N D \_2$ and $N G \_3$ ) in both Osaka and Nagano. As in 2003, a high-isoflavone allele of this QTL was derived from Tamahomare. A QTL located on LG-A1 was detected in Osaka (OT_3,OD_3,OG_3) and Nagano (NGl_4). A highisoflavone allele of this QTL was derived from Peking. QTLs on LG-C1 and LG-D2 were detected only as minor
QTLs for TIC in Osaka and for TIC and GEC in Nagano. In 2004, two QTLs located on LG-A1 and A2 for the isoflavone content were detected together with two minor QTLs located on LG-C1 and D2. The specific QTLs for GLC were also detected on LG-B1 ( $\left.O G l \_4, N G l \_5\right)$.

To evaluate the additive effect of the four QTLs and their interactions, we classified PT-RILs according to genotype of the nearest marker of the four QTLs on LG-A1, A2, C1 and D2, namely Sat_271, I, Satt190 and Satt669. The highisoflavone alleles of these four QTLs were Peking-type, Tamahomare-type, Tamahomare-type and Peking-type, respectively. Two-factor multiple regression analyses were conducted for all combinations of the four QTLs for TIC. In the calculation, the optimum regression model was determined using AIC (Akaike's Information Criterion). The partial regression coefficients for all but the QTL on LG-A1 in Nagano in 2003 and 2004 were significant in all the locations and years tested. Among eight possible pairs of twofactor interactions, only one significant partial regression coefficient was detected for the interaction between the QTLs located on LG-A1 and LG-C1, except in Nag04 (Table 5). To evaluate the relationship between the genotypes of the four QTLs and TIC, we calculated the mean TIC for each genotype group for all locations and years 
Table 3. Effects of QTLs on the total isoflavones content (TIC), total daidzein derivatives content (DAC), total genistein derivatives content (GEC) and total glycitein derivatives content (GLC) detected by means of composite interval mapping in the PT-RIL population planted in Kyoto, Osaka, and Nagano in 2003

\begin{tabular}{|c|c|c|c|c|c|c|c|c|}
\hline Environment & Trait $^{a} \mathrm{mg} / \mathrm{g}$ & QTL & $\mathrm{LG}^{b}$ & Vicinity marker $^{c}$ & Position (cM) & LOD & Additive effect ${ }^{d}$ & Contribution (\%) \\
\hline \multirow[t]{15}{*}{ Kyoto (2003) } & TIC & $K T \_1$ & A2 (8) & Sat_215 & 52.9 & 8.28 & -1.12 & 26.0 \\
\hline & & $K T \_2$ & $\mathrm{C} 1$ (4) & Satt190 & 67.9 & 4.32 & -0.81 & 12.6 \\
\hline & & & D1b (2) & Satt282 & 80.5 & 3.01 & 0.56 & 6.1 \\
\hline & & $K T \_3$ & D2 (17) & Satt669 & 59.0 & 4.16 & 0.67 & 9.3 \\
\hline & DAC & $K D_{-} 1$ & A2 (8) & AW132402 & 63.7 & 9.22 & -0.56 & 20.4 \\
\hline & & & L (19) & Satt373 & 135.3 & 3.42 & 0.33 & 6.2 \\
\hline & GEC & $K G_{-} 1$ & A2 (8) & I & 51.9 & 5.84 & -0.41 & 19.0 \\
\hline & & $K G_{-} 2$ & C1 (4) & Sat_077 & 67.9 & 4.90 & -0.36 & 15.1 \\
\hline & & $K G \_3$ & D2 (17) & Satt669 & 59.0 & 4.96 & 0.27 & 8.1 \\
\hline & GLC & KGl_l & A2 (8) & I & 51.9 & 7.72 & -0.09 & 23.7 \\
\hline & & $K G l_{-} 2$ & B1 (11) & Satt509 & 50.5 & 4.45 & -0.07 & 13.4 \\
\hline & & & B1 (11) & Satt359 & 137.3 & 3.35 & -0.07 & 9.5 \\
\hline & & & D2 (17) & Satt389 & 74.4 & 3.65 & 0.06 & 8.9 \\
\hline & & & G (18) & Sat_143 & 114.6 & 3.07 & 0.05 & 7.5 \\
\hline & & & $\mathrm{H}(12)$ & Satt353 & 6.8 & 3.32 & 0.03 & 2.9 \\
\hline \multirow[t]{11}{*}{ Osaka (2003) } & TIC & $O T \_1$ & A1 (5) & Sat_271 & 104.6 & 4.60 & 0.61 & 7.9 \\
\hline & & $O T \_2$ & A2 (8) & Sat_215 & 52.9 & 5.43 & -0.82 & 12.8 \\
\hline & & & B2 (14) & Satt066 & 73.2 & 3.23 & 0.38 & 3.0 \\
\hline & DAC & $O D \_1$ & A1 (5) & Sat_271 & 104.6 & 4.01 & 0.45 & 13.6 \\
\hline & & $O D \_2$ & A2 (8) & Sat_215 & 52.9 & 5.10 & -0.39 & 9.8 \\
\hline & & & C1 (4) & Sat_042 & 74.3 & 3.48 & -0.39 & 10.0 \\
\hline & GEC & $O G_{-} 1$ & A2 (8) & I & 51.9 & 3.79 & -0.33 & 11.5 \\
\hline & & $O G_{-} 2$ & $\mathrm{C} 1$ (4) & Satt190 & 67.9 & 4.89 & -0.37 & 15.2 \\
\hline & GLC & OGl_l & A2 (8) & I & 51.9 & 4.81 & -0.06 & 15.0 \\
\hline & & $O G l \_2$ & B1 (11) & Satt197 & 62.4 & 8.75 & -0.10 & 38.4 \\
\hline & & & D2 (17) & Sat_092 & 43.5 & 3.45 & 0.06 & 12.8 \\
\hline \multirow[t]{10}{*}{ Nagano (2003) } & TIC & $N T \_1$ & A2 (8) & I & 51.9 & 14.21 & -1.07 & 41.0 \\
\hline & & & $\mathrm{E}(15)$ & Satt213 & 0.0 & 3.05 & -0.27 & 2.7 \\
\hline & DAC & $N D_{-} 1$ & A2 (8) & Sat_215 & 52.9 & 9.36 & -0.50 & 31.5 \\
\hline & & & D2 (17) & Satt669 & 59.0 & 3.18 & 0.30 & 12.0 \\
\hline & GEC & $N G_{-} 1$ & A2 (8) & I & 51.9 & 8.88 & -0.44 & 29.6 \\
\hline & & $N G_{-} 2$ & G (18) & Sat_372 & 157.4 & 4.92 & 0.31 & 15.3 \\
\hline & GLC & $N G l \_1$ & A2 (8) & Sat_215 & 52.9 & 6.99 & -0.06 & 18.6 \\
\hline & & NGl_2 & B1 (11) & Satt197 & 62.4 & 6.83 & -0.07 & 25.7 \\
\hline & & & $\mathrm{C} 1$ (4) & Satt190 & 67.9 & 3.03 & -0.02 & 2.7 \\
\hline & & $N G l \_3$ & C2 (6) & Sat_238 & 116.7 & 7.21 & 0.05 & 11.3 \\
\hline
\end{tabular}

${ }^{a}$ TIC, DAC, GEC, and GLC; see Table 1.

${ }^{b}$ Linkage group (Chromosome number).

${ }^{c}$ Markers located in the vicinity of QTL peaks.

${ }^{d}$ Relative effect of Peking type allele compared with Tamahomare type allele.

(Table 6). TIC had a clear tendency to increase with the number of high isoflavone alleles at four QTLs. Lines harboring Tamahomare alleles at the QTLs located on LG-A1 and LG-C1 exhibited larger TIC values than the lines harboring high-isoflavone alleles at both QTLs, namely, the Peking $(\mathrm{P})$ allele on LG-A1 and the Tamahomare $(\mathrm{T})$ allele on LG-C1. This might be the effect of the interaction between the QTLs located on LG-A1 and LG-C1. Therefore, the line exhibiting the highest isoflavone content harbored a genotype of T, T, T and P-type alleles at the QTLs located on LG-
A1, A2, C1 and D2, respectively. TIC values of 16 different genotypes for the four QTLs were estimated based on multiple linear regressions. As shown in Table 5, all models include the effect of interaction between the QTLs located on LG-A1 and LG-C1 in addition to the effects of the four QTLs. The effects of interactions between the QTLs located on LG-A1 and LG-D2 and those between the QTLs located on LG-C1 and LG-D2 were also added to the multiple linear regression model of Kyo03. The former interaction was also added to the model of Nag03, and the latter interaction was 
Kyoto 2003

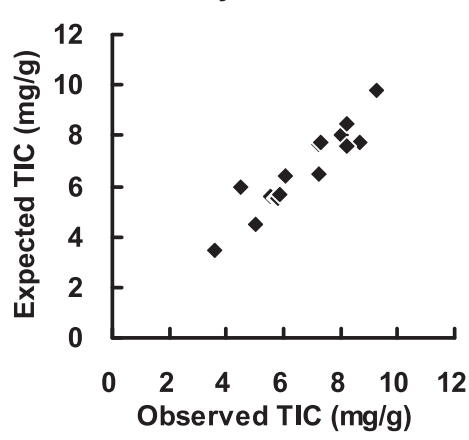

Osaka 2003

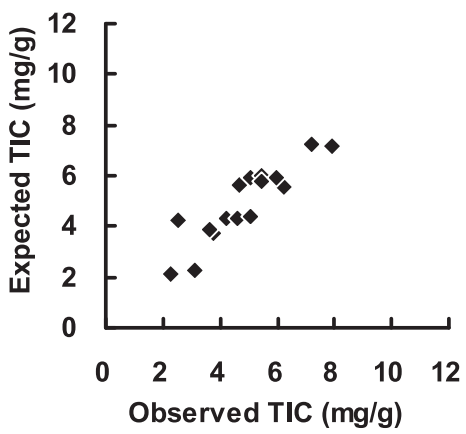

Osaka 2004

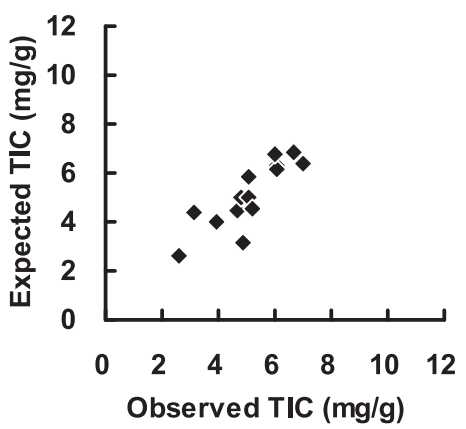

Nagano 2003

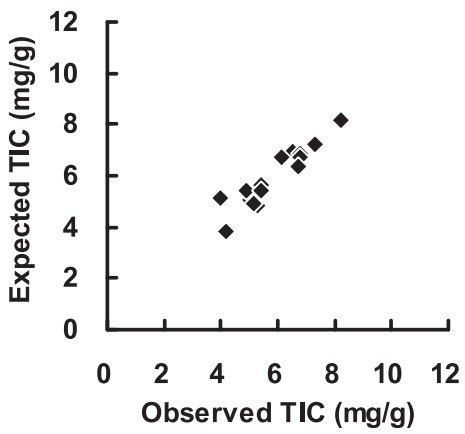

Nagano 2004

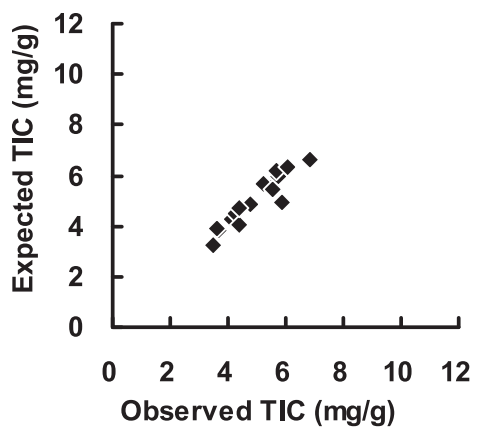

Fig. 3. Relationships between observed and expected total isoflavone content (TIC) values in the sixteen different genotypes for major QTLs controlling TIC of the PT-RIL. The expected TIC values were estimated by multiple linear regression shown in Table 5.

added to the model of Osk03. The expected values of TIC fit to the observed values (Fig. 3), indicating that the four QTLs and their interactions are major factors controlling TIC among PT-RILs. We named the four QTLs on LG- A1 (Chr.5), A2 (Chr.8), C1 (Chr.4) and D2 (Chr.17) qIso1, qIso2, qIso3 and qIso4, respectively.

To evaluate the effects of other agronomic characteristics on isoflavone content, we also conducted QTL analyses for flowering time (S-R1) and maturing time (R1-R8). QTLs $(\mathrm{LOD}>2.5)$ for the flowering time were identified on LGC1 (Chr. 4), C2 (Chr. 6), H (Chr. 12) and O (Chr. 10) in all three locations. Among the QTLs detected on LG-C1, QTLs detected at Nag03 (peak position $71.9 \mathrm{cM})$, Osk04 $(56.5 \mathrm{cM})$ and Nag04 $(69.4 \mathrm{cM})$ are linked to qIso3 and increase the flowering time by harboring a Tamahomare allele. QTLs (LOD > 2.5) for maturing time were identified on LG-B1 (Chr. 11), C1 (Chr. 4), F (Chr. 13) and L (Chr. 19). Among those, QTLs located on LG-C1 detected at Kyo03 (69.4 cM) and Osk03 $(66.3 \mathrm{cM})$ are linked to qIso3 and increase the maturing time by harboring the Tamahomare allele. Therefore, close links between qIso3 and the genetic factors controlling flowering time and/or maturing time might be one reason for the negative correlation between TIC and AMT mentioned in the previous section.

\section{Discussion}

The QTL analysis detected four QTLs for total isoflavone content, which we call qIso1, qIso2, qIso3 and qIso4. The high-isoflavone alleles at qIso1 and qIso 4 were derived from Peking while the high-isoflavone alleles of qIso2 and qIso3 were derived from Tamahomare. The additive effects of the four QTLs that control TIC and their interactions could explain the wide variation in isoflavone content among the PTRILs. TIC ranges reported in previous QTL analyses include $1.9-4.3 \mathrm{mg} / \mathrm{g}$ (Zeng et al. 2009), 1.9-3.7 mg/g (Kasen et al. 2004) and $0.8-3.5 \mathrm{mg} / \mathrm{g}$ (Primomo et al. 2005). The TIC ranges among the PT-RILs in our study were far larger than these: we recorded $2.0-11.0 \mathrm{mg} / \mathrm{g}$ (Kyo03), $0.9-9.9 \mathrm{mg} / \mathrm{g}$ (Osk03), 2.8-9.5 mg/g (Nag03), 1.41-8.69 mg/g (Osk04) and $1.71-8.23 \mathrm{mg} / \mathrm{g}(\mathrm{Nag} 04)$. This indicates that the combination of high-isoflavone alleles derived from distantly related varieties has great potential as a means of controlling the isoflavone content of soybean seeds.

qIsol is located on the LG-A1 (Chr. 5) in the vicinity of Sat_271; nearby, Primomo et al. (2005) have previously reported a QTL controlling TIC, DEC and DAC located in the vicinity of Satt 200 on the same linkage group. According to the integrated map of GmConsensus30_A1 (http:// www.soybase.org/), the map positions of Satt200 and Sat_271 are $92.9 \mathrm{cM}$ and $97.8 \mathrm{cM}$, respectively. As these two QTLs are so close to each other, they are assumed to be the same QTL. Primomo et al. (2005) found that the highisoflavone allele of this QTL is derived from the Canadian variety 'RCAT Angora'. qIso4, which is located on LG-D2 (Chr. 17) in the vicinity of Satt669, has previously been reported by Zeng et al. (2009) as a QTL controlling TIC in the vicinity of Satt208. As indicated in the integrated map of 
Table 4. Effects of QTLs on the total isoflavones content (TIC), total daidzein derivatives content (DAC), total genistein derivatives content (GEC) and total of glycitein derivatives content (GLC) detected by means of composite interval mapping in the PT-RIL population planted in Osaka and Nagano in 2004

\begin{tabular}{|c|c|c|c|c|c|c|c|c|}
\hline Environment & Trait $^{a} \mathrm{mg} / \mathrm{g}$ & QTL & $\mathrm{LG}^{b}$ & Vicinity marker $^{c}$ & Position (cM) & LOD & Additive effect ${ }^{d}$ & Contribution $(\%)$ \\
\hline \multirow[t]{12}{*}{ Osaka (2004) } & \multirow[t]{4}{*}{ TIC } & $O T \_3$ & A1 (5) & Sat_271 & 104.64 & 4.11 & 0.63 & 11.5 \\
\hline & & $O T_{-} 4$ & A2 (8) & I & 51.86 & 7.32 & -0.86 & 21.4 \\
\hline & & & C1 (4) & Sat_042 & 74.26 & 3.00 & -0.44 & 5.9 \\
\hline & & & D1b (2) & Satt546 & 101.38 & 3.01 & 0.17 & 0.9 \\
\hline & \multirow[t]{3}{*}{$\mathrm{DAC}$} & $O D \_3$ & A1 (5) & Sat_271 & 104.64 & 7.96 & 0.51 & 24.5 \\
\hline & & $O D \_4$ & A2 (8) & AW132402 & 63.74 & 6.55 & -0.41 & 15.6 \\
\hline & & $O D \_5$ & $\mathrm{E}(15)$ & Satt384 & 16.98 & 3.91 & -0.04 & 0.2 \\
\hline & \multirow[t]{2}{*}{ GEC } & $O G \_3$ & A1 (5) & Satt200 & 98.31 & 4.50 & 0.20 & 7.2 \\
\hline & & $O G_{-} 4$ & A2 (8) & I & 51.86 & 4.66 & -0.30 & 15.9 \\
\hline & \multirow[t]{3}{*}{ GLC } & $O G l \_3$ & A2 (8) & I & 51.86 & 5.38 & -0.07 & 15.4 \\
\hline & & $O G l \_4$ & B1 (11) & Satt197 & 62.39 & 6.75 & -0.08 & 24.0 \\
\hline & & & $\mathrm{G}(18)$ & Sat_372 & 157.39 & 3.55 & 0.05 & 9.5 \\
\hline \multirow[t]{12}{*}{ Nagano (2004) } & \multirow[t]{2}{*}{ TIC } & \multirow[t]{2}{*}{$N T_{\_} 2$} & A2 (8) & AW132402 & 63.74 & 7.75 & -0.79 & 26.0 \\
\hline & & & D2 (17) & Satt669 & 59.02 & 3.67 & 0.48 & 9.7 \\
\hline & \multirow[t]{3}{*}{ DAC } & $N D \_2$ & A2 (8) & AW132402 & 63.74 & 9.17 & -0.39 & 21.9 \\
\hline & & $N D \_3$ & F (13) & Satt114 & 89.62 & 3.74 & 0.29 & 13.1 \\
\hline & & & L (19) & Satt373 & 135.32 & 3.11 & 0.13 & 2.6 \\
\hline & \multirow[t]{3}{*}{ GEC } & \multirow[t]{3}{*}{$N G \_3$} & A2 (8) & AW132402 & 63.74 & 5.31 & -0.32 & 20.0 \\
\hline & & & D2 (17) & Satt669 & 59.02 & 3.50 & 0.19 & 7.1 \\
\hline & & & K (9) & Sat_119 & 8.86 & 3.06 & 0.17 & 5.2 \\
\hline & \multirow[t]{4}{*}{ GLC } & \multirow[t]{2}{*}{ NGl_4 } & A1 (5) & Satt276 & 21.39 & 3.86 & 0.04 & 10.1 \\
\hline & & & A2 (8) & Sat_215 & 52.86 & 3.41 & -0.03 & 7.5 \\
\hline & & \multirow[t]{2}{*}{$N G l \_5$} & B1 (11) & Satt197 & 62.39 & 10.19 & -0.07 & 37.4 \\
\hline & & & C2 (6) & Sat_238 & 116.67 & 3.25 & 0.03 & 6.6 \\
\hline
\end{tabular}

GmConsensus30_D2 (http://www.soybase.org/), Satt669 $(67.7 \mathrm{cM})$ and Satt208 $(67.9 \mathrm{cM})$ are closely linked. Zeng et al. found that the high-isoflavone allele of this QTL is derived from the Chinese variety 'Zhongdou27'. Thus it appears that high-isoflavone alleles derived from Peking may be present at qIsol and qIso4 in many soybean varieties.

In contrast to qIsol and qIso4, no previous report has mentioned QTLs in the vicinity of qIso2 and qIso3. Therefore, these high-isoflavone alleles, which are derived from the Japanese variety Tamahomare, are considered to be novel QTLs controlling isoflavone content. It is reasonable to assume that the other Japanese high-isoflavone soybean varieties harbor the same allele as Tamahomare does. To clarify the distribution of the high-isoflavone alleles at qIso2 and $q I s o 3$ among Japanese varieties, segregation analysis is needed for isoflavone content using progeny lines derived from crosses between Tamahomare and other Japanese varieties. Efficient genotypic analyses and association analyses will become available with the development of molecular markers for qIso2 and qIso3. The results obtained in this study indicate that the effects of the qIso2 and qIso3 allele on isoflavone content are much more stable under different environmental conditions than those of qIsol and qIso4 are. Our findings will therefore contribute significantly to the development of molecular markers for use in breeding both high-isoflavone and low-isoflavone varieties.

qIso2 controls not only the total isoflavone content but also the content of three major isoflavone groups. This QTL is very stable under different environmental conditions, as seen through our experiments in different years with different temperatures: it exhibited large LOD scores for TIC, DAC, GEC and GLC in all environments. We therefore consider qIso2 to be the most significant QTL controlling the levels of all isoflavone groups. The function of qIso2 might be related to the up-regulation of upstream enzymes of the phenylpropanoid pathway, including phenylalanine ammonia-lyase (PAL) and chalcone synthase (CHS), which might increase the levels of all isoflavone groups, as Yu et al. (2003) have suggested. Recently, Dhaubhadel et al. (2007) demonstrated that higher-TIC varieties exhibited a stronger expression of $\mathrm{CHS7}$ and $\mathrm{CHS} 8$, indicating that these two genes play a crucial role in isoflavone synthesis. qIso2 was detected in the vicinity of the $I$ locus, where three $C H S$ genes, CHS1, CHS3 and CHS4 are located in tandem (Todd 
Table 5. Partial regression coefficients of four QTLs and their twofactor interactions on the total isoflavone contents in PT-RILs calculated from the linear multiple regression models selected by Akaike's information criterion (AIC) ${ }^{a}$

\begin{tabular}{|c|c|c|c|c|c|}
\hline Coefficient & $\begin{array}{c}\text { Kyoto } \\
2003\end{array}$ & $\begin{array}{l}\text { Osaka } \\
2003\end{array}$ & $\begin{array}{c}\text { Nagano } \\
2003\end{array}$ & $\begin{array}{l}\text { Osaka } \\
2004\end{array}$ & $\begin{array}{c}\text { Nagano } \\
2004\end{array}$ \\
\hline Interception & $6.641 * * *$ & $4.907 * * *$ & $6.027 * * *$ & $5.043 * * *$ & $4.995 * * *$ \\
\hline A1 & $0.373 *$ & $0.531 * *$ & 0.036 & $0.554 * * *$ & 0.088 \\
\hline $\mathrm{A} 2$ & $-1.031 * * *$ & $-0.804 * * *$ & $-0.904 * * *$ & $-0.910 * * *$ & $-0.823 * * *$ \\
\hline $\mathrm{C} 1$ & $-0.727 * * *$ & $-0.875 * * *$ & $-0.325 *$ & $-0.370 * *$ & $-0.316^{*}$ \\
\hline D2 & $0.421 *$ & $0.334 *$ & $0.449 * *$ & $0.276 *$ & $0.334 * *$ \\
\hline \multicolumn{6}{|l|}{$\overline{A 1: A 2}$} \\
\hline $\mathrm{A} 1: \mathrm{C} 1$ & $0.665 * * *$ & $0.504 * *$ & $0.288 *$ & $0.326^{*}$ & 0.228 \\
\hline A1:D2 & -0.261 & & -0.191 & & \\
\hline \multicolumn{6}{|l|}{$\mathrm{A} 2: \mathrm{C} 1$} \\
\hline \multicolumn{6}{|l|}{ A2:D2 } \\
\hline $\mathrm{C} 1: \mathrm{D} 2$ & -0.281 & -0.279 & & & \\
\hline Joint F-test & $14.04 * * *$ & $15.30 * * *$ & $11.77 * * *$ & $22.46 * * *$ & $12.79 * * *$ \\
\hline adjusted $\mathrm{R}^{2 b}$ & $\begin{array}{c}0.490 \\
(0.413)\end{array}$ & $\begin{array}{c}0.480 \\
(0.434)\end{array}$ & $\begin{array}{c}0.415 \\
(0.386)\end{array}$ & $\begin{array}{c}0.538 \\
(0.508)\end{array}$ & $\begin{array}{c}0.388 \\
(0.371)\end{array}$ \\
\hline
\end{tabular}

$*, * *, * * *$ : Significant at $5 \%, 1 \%$ and $0.1 \%$, respectively.

${ }^{a}$ Partial regression coefficients are shown for variables selected by AIC.

${ }^{b}$ In parensis, adjusted $\mathrm{R}^{2}$ values of the linear multiple regression models without including two-factor interaction.

and Vodkin 1996, Senda et al. 2002, Senda et al. 2004, Clojgh et al. 2004). However, there is no significant difference in the expression levels of CHS1, CHS3 and CHS4 at the mature stage between PT-RILs with high isoflavone con- tent and those with low isoflavone content (data not shown). Furthermore, our measurements of TIC in Japanese soybean varieties revealed no significant difference in TIC between the yellow seed coat group (II) and the black seed coat group (ii). This indicates that $q I$ so 2 and $I$ are not allelic. Based on the genome database of soybean variety Williams82 (http:// www.phytozome.net/), there are several protein kinases and transcription factors in the vicinity of $I$. Further studies should be done to disclose the relationships between those transcription factors and qIso2.

The second most significant QTL, qIso3, was involved in TIC and GEC in Kyoto, DAC and GEC in Osaka and GLC in Nagano. Effects of interaction between qIso1 and qIso3 have been detected. When both QTLs harbor Tamahomaretype alleles, their interactions increase TIC. Interestingly, the interaction of qIso1 and qIso3 was significant even under environmental conditions in which the main effect of qIsol was not significant (Nag03, Table 5).

In addition to the four QTLs associated with TIC, we also identified a QTL associated with GLC on LG-B1 (Chr. 11) in the vicinity of Satt197. Meksem et al. (2001) and Kassem et al. (2004) have also reported QTLs associated with GLC in the vicinity of Satt197 and Satt251, respectively. This suggests that the QTL specific to GLC is also broadly distributed among soybean varieties. Wang et al. (1994b) have demonstrated that the glycitein content in soybean seed is more stable across different environmental conditions than the daidzein and genistein levels are. Furthermore, the correlation coefficient between TIC and GLC is smaller than that between TIC and DAC and that between TIC and GEC,

Table 6. Relationships between the genotypes at four QTLs for isoflavone content and the mean total isoflavone content of PT-RILs planted in Kyoto, Osaka and Nagano in 2003 and 2004

\begin{tabular}{|c|c|c|c|c|c|c|c|c|c|c|}
\hline \multirow[b]{2}{*}{$\begin{array}{l}\text { No of high- } \\
\text { isoflavone } \\
\text { alleles }\end{array}$} & \multicolumn{4}{|c|}{ "Genotype of four QTL alleles ${ }^{a}$} & \multicolumn{5}{|c|}{ "Mean TIC of each genotype group } & \multirow[b]{2}{*}{$\begin{array}{l}\text { Total } \\
\text { mean }\end{array}$} \\
\hline & $\begin{array}{c}\text { LG-A }^{b} \\
\text { Chr.5 } \\
\text { Sat_271 }\end{array}$ & $\begin{array}{c}\text { LG-A2: } \\
\text { Chr.8 } \\
\text { I }\end{array}$ & $\begin{array}{c}\text { LG-C1: } \\
\text { Chr.4 } \\
\text { Satt190 }\end{array}$ & $\begin{array}{c}\text { LG-D2: } \\
\text { Chr.17 } \\
\text { Satt669 }\end{array}$ & $\begin{array}{c}\text { Kyoto } \\
2003\end{array}$ & $\begin{array}{c}\text { Osaka } \\
2003\end{array}$ & $\begin{array}{c}\text { Nagano } \\
2003\end{array}$ & $\begin{array}{c}\text { Osaka } \\
2004\end{array}$ & $\begin{array}{c}\text { Nagano } \\
2004\end{array}$ & \\
\hline 0 & & & & & 3.58 & 2.27 & 4.16 & 2.62 & 3.49 & 3.22 \\
\hline \multirow[t]{4}{*}{1} & & & & + & 4.49 & 2.52 & 5.28 & 3.10 & 3.69 & 3.82 \\
\hline & + & & & & 5.04 & 3.08 & 3.96 & 4.89 & 3.60 & 4.12 \\
\hline & & & + & & 5.56 & 4.20 & 5.02 & 3.94 & 4.12 & 4.57 \\
\hline & & + & & & 5.77 & 3.78 & 5.39 & 4.66 & 4.78 & 4.88 \\
\hline \multirow[t]{6}{*}{2} & + & & & + & 5.82 & 4.59 & 4.92 & 4.80 & 4.27 & 4.88 \\
\hline & + & & + & & 5.89 & 5.08 & 5.15 & 4.69 & 4.37 & 5.04 \\
\hline & & + & & + & 7.22 & 3.63 & 6.52 & 5.08 & 5.50 & 5.59 \\
\hline & & + & + & & 7.23 & 5.04 & 6.77 & 5.09 & 5.76 & 5.98 \\
\hline & + & + & & & 8.02 & 5.43 & 6.77 & 6.04 & 5.53 & 6.36 \\
\hline & & & + & + & 8.68 & 6.20 & 6.74 & 5.22 & 5.86 & 6.54 \\
\hline \multirow[t]{4}{*}{3} & + & & + & + & 6.09 & 4.68 & 5.38 & 4.78 & 4.41 & 5.07 \\
\hline & + & + & + & & 7.34 & 5.47 & 6.13 & 6.05 & 5.26 & 6.05 \\
\hline & + & + & & + & 8.19 & 5.95 & 7.37 & 5.97 & 5.68 & 6.63 \\
\hline & & + & + & + & 9.27 & 7.94 & 8.23 & 7.02 & 6.86 & 7.86 \\
\hline 4 & + & + & + & + & 8.23 & 7.19 & 7.30 & 6.67 & 6.06 & 7.09 \\
\hline
\end{tabular}

$a+$ indicates high isoflavone allelles derived from Peking and Tamahomare.

${ }^{b}$ Linkage groups, chromosome numbers and the vicinity markers of QTL are shown in top, middle and bottom rows, respectively. 
indicating that GLC is governed by one or more specific genetic factors not involved in TIC. It is known that glycitein derivatives are synthesized via a biosynthetic pathway different from those of daidzein and genistein derivatives, a pathway that involves the synthesis of liquiritigenin and chalcone. In the biosynthetic branch of glycitein derivatives, 6,7,4'-trihydroxy flavanone is first synthesized from liquiritigenin by the $6^{\prime}$-hydroxylation of the A ring, which is catalyzed by oxidoreductase (F6H) (KEGG; http://www. genome.jp/kegg/pathway/map/map00943.html). Since this QTL was not responsible for TIC in any environment, it is considered to act specifically on glycitein precursors in this branch. Further analysis of the activities of enzymes involved in this branch would provide novel insight into the function of this QTL.

In this paper, we have identified two novel QTLs associated with total isoflavone content and shown that these QTLs are stable by detecting them in multiple years and under multiple temperature conditions. These findings will contribute to the development of marker-assisted selection for isoflavone content and to the identification of the genes contributing to the physiological process of isoflavone accumulation in soybean seeds.

\section{Literature Cited}

Alekel,D.L., A.St.Germain, C.T.Peterson, K.B.Hanson, J.W.Stewart and T.Toda (2000) Isoflavone-rich soy protein isolate attenuates bone loss in the lumbar spine of perimenopausal women. Am. J. Clin. Nutr. 72: 844-852.

Barz,W. and R. Welle (1992) Biosynthesis and metabolism of isoflavones and pterocarpan phytoalexins in chickpea, soybean and phytopathogenic fungi. In: Stafford,H.A. and R.K.Ibrahim (eds.) Recent Advances in Phytochemistry, Vol. 26, Plenum Press, New York, pp. 139-164.

Clough, S.J., J.H. Tuteja， M.Li， L.F.Marek, R.C. Shoemaker and L.O.Vodkin (2004) Features of a 103-kb gene-rich region in soybean include an inverted perfect repeat cluster of CHS genes comprising the $I$ locus. Genome 47: 819-831.

Dhaubhadel,S., M.Gijzen, P.Moy and M.Farhangkhoee (2007) Transcriptome analysis reveals a critical role of $C H S 7$ and $C H S 8$ genes for isoflavonoid synthesis in soybean seeds. Plant Physiol. 143: 326-338.

Dixon, R.A., M.J.Harrison and N.L.Paiva (1995) The isoflavonoid phytoalexin pathway: From enzymes to genes to transcription factors. Physiol. Plantarum 93: 385-392.

Dixon, R.A. and N.L.Paiva (1995) Stress-induced phenylpropanoid metabolism. Plant Cell 7: 1085-1097.

Fitzpatrick, M. (1998) Comments on isoflavones in soy-based infant formulas. J. Agr. Food Chem. 46: 3396-3397.

Graham,T.L. and M.Y.Graham (1996) Signaling in soybean phenylpropanoid responses. Plant Physiol. 110: 1123-1133.

Kassem,M.A., K. Meksem, M.J.Iqbal, V.N.Njiti, W.J.Banz, T.A. Winters, A.Wood and D.A.Lightfoot (2004) Definition of soybean genomic regions that control seed phytoestrogen amounts. J. Biomed. Biotechnol. 1: 52-60.

Kassem,M.A., J.Shultz, K.Meksem, Y.Cho, A.J.Wood, M.J.Iqbal and D.A.Lightfoot (2006) An updated 'Essex' by 'Forrest' linkage map and first composite interval map of QTL underlying six soybean traits. Theor. Appl. Genet. 113: 1015-1026.

Kudou,S., Y.Fleury, D.Welti, D.Magnolato, T.Uchida and K.Kitamura (1991) Malonyl isoflavone glycosides in soybean seeds (Glycine max MERRILL). Agr. Biol. Chem. 55: 2227-2233.

Landini,S., M.Y.Graham and T.L.Graham (2002) Lactofen induces isoflavone accumulation and glyceollin elicitation competency in soybean. Phytochemistry 62: 865-874.

Lissin,L.W. and J.P.Cooke (2000) Phytoestrogens and cardiovascular health. J. Am. Coll. Cardiol. 35: 1403-1410.

Mackenbrock, U., R.Vogelsang and W.Barz (1992) Isoflavone and pterocarpan malonylglucosides and $\beta$-1,3-glucan- and chitinhydrolases are vacuolar constituents in chickpea (Cicer arietinum L.). Z. Naturforsch. 47c: 815-822.

McIntosh, M.F. (1983) Analysis of combined experiments. Agron. J. 75: $153-155$.

Meksem,K., V.N.Njiti，W.J.Banz，M.J.Iqbal，M.M.Kassem, D.L. Hyten, J.Yuang, T.A.Winters and D.A.Lightfoot (2001) Genomic regions that underlie soybean seed isoflavone content. J. Biomed. Biotechnol. 1: 38-44.

MerzDemlow, B.E., A.M.Duncan, K.E. Wangen, X.Xu, T.P.Carr, W.R.Phipps and M.S.Kurzer (2000) Soy isoflavones improve plasma lipids in normocholesterolemic, premenopausal women. Am. J. Clin. Nutr. 71: 1462-1469.

Primomo, V.S., V.Poysa, G.R.Ablett, C.J.Jackson, M.Gijzen and I.Rajcan (2005) Mapping QTL for individual and total isoflavone content in soybean seeds. Crop Sci. 45: 2454-2464.

Pueppke,J.L. (1996) The genetic and biochemical basis for nodulation of legumes by rhizobia. Crit. Rev. Biotechnol. 16: 1-51.

Rivera-Vargas,L.I., A.F.Schmitthenner and T.L.Graham (1993) Soybean flavonoid effects on and metabolism by Phytophthora sojae. Phytochemistry 32: 851-857.

van Rhijn,P. and J.Vanderleyden (1995) The Rhizobium-plant symbiosis. Microbiol. Rev. 59: 124-142.

Sayama,T., T.Nakazaki, G. Ishikawa, K. Yagasaki, N. Yamada, N. Hirota, K. Hirata, T. Yoshikawa, M.Teraishi, Y.Okumoto, T. Tsukiyama and T.Tanisaka (2009) QTL analysis of seed-flooding tolerance in soybean (Glycine $\max$ (L.) Merr.). Plant Sci. 176: 514521.

Senda,M., A.Kasai, S.Yumoto, S.Akada, R.Ishikawa, T.Harada and M.Niizeki (2002) Sequence divergence at chalcone synthase gene in pigmented seed coat soybean mutants of the Inhibitor locus. Gene. Genet. Syst. 77: 341-350.

Senda,M., C.Masuta, S.Ohnishi, K.Goto, A.Kasai, T.Sano, J.S.Hong and S.MacFarlane (2004) Patterning of virus-infected Glycine max seed coat is associated with suppression of endogenous silencing of chalcone synthase genes. Plant Cell 16: 807-818.

Setchell, K.D. (2001) Soy isoflavones-benefits and risks from nature's selective estrogen receptor modulators (SERMs). J. Am. Coll. Nutr. 20: 354S-362S.

Setchell,K.D., L.Zimmer-Nechemias, J.Cai and J.E.Heubi (1997) Exposure of infants to phyto-oestrogens from soy-based infant formula. Lancet 350: 23-27.

Todd,J.J. and L.O.Vodkin (1996) Duplications that suppress and deletions that restore expression from a chalcone synthase multigene family. Plant Cell 8: 687-699.

Tsukamoto, C., S.Shimada, K.Igita, S.Kudou, M.Kokubun, K.Okubo and K.Kitamura (1995) Factors affecting isoflavone content in soybean seeds: changes in isoflavones, saponins, and composition of fatty acids at different temperatures during seed development. J. Agr. Food Chem. 43: 1184-1192.

Uesugi,T., T.Toda, K.Tsuji and H.Ishida (2001) Comparative study on 
reduction of bone loss and lipid metabolism abnormality in ovariectomized rats by soy isoflavones, daidzin, genistin, and glycitin. Biol. Pharmceut. Bull. 24: 368-372.

Vyn, T.J., X.Yin, T.W.Bruulsema, C.J.Jackson, I.Rajcan and S.M. Brouder (2002) Potassium fertilization effects on isoflavone concentrations in soybean. J. Agr. Food Chem. 50: 3501-3506.

Wang,H.J. and P.A.Murphy (1994a) Isoflavone content in commercial soybean foods. J. Agr. Food Chem. 42: 1666-1673.

Wang,H. and P.A.Murphy (1994b) Isoflavone composition of American and Japanese soybeans in Iowa: effects of variety, crop year, and location. J. Agr. Food Chem. 42: 1674-1677.

Watanabe, S., T.Tajuddin, N.Yamanaka, M.Hayashi and K.Harada (2004) Analysis of QTLs for reproductive development and seed quality traits in soybean using recombinant inbred lines. Plant Breed. 54: 399-407.

Yellayi, S., A.Naaz, M.A.Szewczykowski, T.Sato, J.A. Woods, J. Chang, M. Segre, C.D.Allred, W.G. Helferich and P.S.Cooke (2002) The phytoestrogen genistein induces thymic and immune changes: A human health concern? Proc. Natl. Acad. Sci. USA 99: 7616-7621.

Yoshikawa,T., Y.Okumoto, M.Terai, K.Yamada, M.Teraishi, T. Tsukiyama and T.Tanisaka (2009) High isoflavone content mutants induced from the soybean variety 'Tambaguro' with gamma irradiation. J. Crop. Res. 54: 111-118.

Yu,O., J.Shi, A.O.Hession, C.A.Maxwell, B.McGonigle and J.T. Odell (2003) Metabolic engineering to increase isoflavone biosynthesis in soybean seed. Phytochemistry 63: 753-763.

Zeng,Z.B. (1993) Theoretical basis for separation of multiple linked gene effects in mapping quantitative trait loci. Proc. Natl. Acad. Sci. USA 90: 10972-10976.

Zeng,Z.B. (1994) Precision mapping of quantitative trait loci. Genetics 136: $1457-1468$

Zeng,G., D.Li, Y.Han, W.Teng, J.Wang, L.Qiu and W.Li (2009) Identification of QTL underlying isoflavone contents in soybean seeds among multiple environments. Theor. Appl. Genet. 118: $1455-1463$. 